\title{
PROFILE OF PEDIATRIC SOLID TUMOR CARE AND MULTIDISCIPLINARY TUMOR BOARDS IN SOUTHEAST ASIA
}

\author{
Mohd Yusran Othman ${ }^{1}$, Sally Blair ${ }^{2}$, Shireen Anne Nah ${ }^{3}$, Hany Ariffin ${ }^{3}$, Chatchawin \\ Assanasen ${ }^{4}$, Shui Yen Soh ${ }^{5}$, Anette S Jacobsen ${ }^{5}$, Catherine Lam ${ }^{6}$, and Amos Loh ${ }^{5}$ \\ ${ }^{1}$ Hospital Tunku Azizah (Kuala Lumpur Women and Children Hospital) \\ ${ }^{2}$ Vietnam Pediatric Hematology Oncology (PHO) Programme \\ ${ }^{3}$ University of Malaya \\ ${ }^{4}$ UT Health San Antonio \\ ${ }^{5}$ KK Women's and Children's Hospital \\ ${ }^{6}$ St. Jude Children's Research Hospital
}

May 11, 2020

\begin{abstract}
Background Pediatric solid tumors require coordinated multidisciplinary specialist care. However, expertise and resources to conduct multidisciplinary tumor board (MDTB) meetings are lacking in low- and middle-income countries (LMICs). We aimed to profile practices and perceptions on MDTBs among pediatric solid tumor units (PSTUs) in Southeast Asian LMIC countries. Methods Using online survey forms, availability of specialty manpower and MDTBs among PSTUs was first determined. From the subset of PSTUs with MDTBs, 1 pediatric surgeon and 1 pediatric oncologist from each center were queried using 5-point Likert scale questions adapted from published questionnaires. Results In 37/46 (80.4\%) identified PSTUs, pediatric-trained oncologists, surgeons, radiologists, pathologists, radiation oncologists, nuclear medicine physicians and nurses were available in $94.6 \%, 91.9 \%, 54.1 \%, 40.5 \%, 29.7 \%, 13.5 \%$ and $81.1 \%$ of PSTUs, respectively. Availability of pediatric-trained surgeons, radiologists and pathologists were significantly associated with existence of MDTBs ( $\mathrm{p}=0.037,0.005,0.022$ respectively). Among $43 / 48(89.6 \%)$ respondents from 24 PSTUs with MDTBs, $90.5 \%$ of oncologists reported $>50 \%$ oncology-dedicated workload versus $22.7 \%$ of surgeons. Views on benefits and barriers did not significantly differ between both groups. Majority agreed MDTBs helped improve accuracy of treatment recommendations and team competence. Complex cases, insufficient radiology and pathology preparation, and need for supplementary investigations, were the top barriers. Conclusions Availability of pediatric-trained subspecialists was a significant prerequisite for pediatric MDTBs. Most PSTUs lacked pediatric-trained pathologists and radiologists. Correspondingly, gaps in radiographic and pathological diagnoses were the commonest limitations. Greater emphasis on holistic multidisciplinary subspecialty development is needed to advance pediatric solid tumor care in Southeast Asia.
\end{abstract}

Profile of Pediatric Solid Tumor Care and Multidisciplinary Tumor Boards in Southeast Asia Mohd Yusran Othman ${ }^{1,2}$, Sally Blair ${ }^{3,4}$, Shireen A Nah ${ }^{5}$, Hany Ariffin ${ }^{6}$, Chatchawin Assanasen ${ }^{3,7}$, Shui Yen $\mathrm{Soh}^{8}$, Anette S Jacobsen ${ }^{1,9}$, Catherine Lam $^{10}$, Amos HP Loh ${ }^{1,9}$ *

${ }^{1}$ Department of Pediatric Surgery, KK Women's and Children's Hospital, Singapore

${ }^{2}$ Department of Pediatric Surgery, Hospital Tunku Azizah (Women and Children Hospital), Kuala Lumpur, Malaysia 
${ }^{3}$ Vietnam Pediatric Hematology Oncology (PHO) Programme, Ho Chi Minh City, Vietnam

${ }^{4}$ Division of Pediatric Haematology and Oncology, Department of Pediatrics, National University of Singapore, Singapore

${ }^{5}$ Division of Pediatric Surgery, Department of Surgery, Faculty of Medicine, University of Malaya, Malaysia

${ }^{6}$ Department of Pediatrics, Faculty of Medicine, University of Malaya, Malaysia

7 SEAPHO, Division of Hematology/Oncology Department of Pediatrics, UT Health San Antonio, San Antonio, Texas

8 Haematology/Oncology Service, Department of Pediatric Subspecialties, KK Women's and Children's Hospital, Singapore

${ }^{9}$ SingHealth-Duke NUS Global Health Institute, Duke NUS Medical School, Singapore

10 St Jude Global, St Jude Children's Research Hospital, Memphis, Tennessee

*Correspondence: Dr Amos Loh, Department of Pediatric Surgery, KK Women's and Children's Hospital, 100 Bukit Timah Road, Singapore 299899, Singapore. Tel: +65-63948014, Email: amos.loh.h.p@singhealth.com.sg

Text word count: 2830;

Abstract word count: 250;

Brief running title: Pediatric solid tumor boards in Southeast Asia

Key words: Pediatric solid tumors, multidisciplinary tumor board, Southeast Asia, Pediatric Oncology in Developing Countries (PODC)

Tables: 2

Figures: 3

Supporting Information files: 4

\begin{tabular}{ll}
\hline Abbreviations & Abbreviations \\
\hline ASEAN & Association of Southeast Asian Nations \\
CPD & Continuing Professional Development \\
GNI & gross national income \\
LMIC & multidisciplinary tumor board \\
MDTB & Pediatric Cancer Unit \\
PCU & Paediatric Oncology in Developing Countries \\
PODC & pediatric solid tumor unit \\
PSTU & Southeast Asia Pediatric Hematology Oncology \\
SEAPHO & International Society of Paediatric Oncology \\
SIOP & World Health Organization \\
WHO &
\end{tabular}

\section{Meeting abstract}

The abstract has been submitted for oral presentation for the $52^{\text {nd }}$ Annual Congress of the International Society of Paediatric Oncology (SIOP 2020) that will take place in Ottawa, Canada, October 14 to 17, 2020 (pending approval).

\section{Abstract}




\section{Background}

Pediatric solid tumors require coordinated multidisciplinary specialist care. However, expertise and resources to conduct multidisciplinary tumor board (MDTB) meetings are lacking in low- and middle-income countries (LMICs). We aimed to profile practices and perceptions on MDTBs among pediatric solid tumor units (PSTUs) in Southeast Asian LMIC countries.

\section{Methods}

Using online survey forms, availability of specialty manpower and MDTBs among PSTUs was first determined. From the subset of PSTUs with MDTBs, 1 pediatric surgeon and 1 pediatric oncologist from each center were queried using 5-point Likert scale questions adapted from published questionnaires.

\section{Results}

In 37/46 (80.4\%) identified PSTUs, pediatric-trained oncologists, surgeons, radiologists, pathologists, radiation oncologists, nuclear medicine physicians and nurses were available in $94.6 \%, 91.9 \%, 54.1 \%, 40.5 \%$, $29.7 \%, 13.5 \%$ and $81.1 \%$ of PSTUs, respectively. Availability of pediatric-trained surgeons, radiologists and pathologists were significantly associated with existence of MDTBs $(p=0.037,0.005,0.022$ respectively). Among 43/48 (89.6\%) respondents from 24 PSTUs with MDTBs, 90.5\% of oncologists reported $>50 \%$ oncology-dedicated workload versus $22.7 \%$ of surgeons. Views on benefits and barriers did not significantly differ between both groups. Majority agreed MDTBs helped improve accuracy of treatment recommendations and team competence. Complex cases, insufficient radiology and pathology preparation, and need for supplementary investigations, were the top barriers.

\section{Conclusions}

Availability of pediatric-trained subspecialists was a significant prerequisite for pediatric MDTBs. Most PSTUs lacked pediatric-trained pathologists and radiologists. Correspondingly, gaps in radiographic and pathological diagnoses were the commonest limitations. Greater emphasis on holistic multidisciplinary subspecialty development is needed to advance pediatric solid tumor care in Southeast Asia.

\section{Introduction}

Southeast Asia has been the scene of substantial initiatives in pediatric cancer resource development in recent years ${ }^{1-4}$. With a total population of 668 million, the region is home to $8.5 \%$ of the global childhood population aged 14 and under, with approximately 16,000 new cases of childhood cancer annually and the $3^{\text {rd }}$ highest rate of childhood cancer mortality worldwide, after Western and North Africa ${ }^{5-7}$. Although 9 of the 11 countries that comprise the region are low- and middle-income countries (LMICs) ${ }^{8}$, advancements have been achieved in treatment standards and outcomes in pediatric cancer, yet significant gaps still remain particularly with respect to the care of solid and brain tumors ${ }^{9-16}$.

Solid tumor management requires the coordinated effort of teams of multiple medical specialties, and varied infrastructural resources ranging from surgical and radiation facilities to laboratory and pathology services 17. Interestingly, while availability of each of these elements may vary between centers, this does not preclude delivery of effective curative treatment for pediatric tumors when available resources can be appropriately channeled ${ }^{18,19}$. This underscores the importance of the multidisciplinary tumor board (MDTB) as a critical element for advancing pediatric solid tumor care, and one that remains relevant even in LMICs ${ }^{20-22}$. However, organizing MDTBs can be an organizational burden and amounts to extra workload for the involved personnel 23,24 , especially in centers with already-limited resources.

From a pilot survey of pediatric surgeons in Southeast Asia, we found that not all centers in Southeast Asia that care for childhood tumor patients had pediatric MDTBs, and in centers that had them, pediatric oncologists and surgeons were the two specialists that were most involved in these meetings. Hence, we conducted a cross-sectional survey to profile MDTBs from pediatric oncology centers in LMIC countries around Southeast Asia, and to study perceptions on benefits and barriers for MDTBs among pediatric surgeons and pediatric oncologists. 


\section{Methods}

\subsection{Participants and definitions}

Potential survey centers and participants were identified through membership records of regional professional associations and scientific meetings, particularly the St. Jude-VIVA Forum in Pediatric Oncology and ASEAN Society of Pediatric Surgeons. This research study (SHS/CIRB/2020/2020) was granted IRB waiver.

We defined pediatric solid tumor units (PSTUs) as institutional departments that care for pediatric solid tumors, with at least 1 pediatric oncologist or 1 pediatric surgeon who are either in-house or employed in a part-time capacity. Multidisciplinary tumor boards (MDTBs) were defined as any formal meeting attended by at least pediatric oncologists and pediatric surgeons, together with one more related subspecialty (pediatric-trained or general radiologists, radiation oncologists, pathologists, nuclear medicine physicians and nurses).

For the first part of the study, we included all PSTUs from Southeast Asian LMICs with at least one respondent (either pediatric oncologist or pediatric surgeon) in order to profile the available specialties and MDTBs at each PSTU. Exclusion criteria were refusal to participate or non-response, and incomplete or delayed responses beyond study period. For the survey in the second part of the study, only PSTUs with MDTBs were involved.

\section{Development and conduct of survey}

From the 46 Southeast Asian PSTUs, centers with MDTBs were shortlisted for the full survey. From this subset of PSTUs with MDTBs, 1 pediatric surgeon and 1 pediatric oncologist from each center were contacted via email to complete the survey via an online electronic form or a manual form. Automatic online language translation was used in the former to assist with respondents with difficulty with English language. Following two weeks given for response, a second oncologist or surgeon from the PSTU was contacted, failing which a null response was recorded.

Survey questions were adapted from published surveys on MDTB organization and dynamics ${ }^{23-25}$, and drafted in English. The survey was composed of three main parts. First, to profile the respondents, they were asked about their type of specialty, years of practice and their estimated oncology workload. Next, to profile the center's MDTB, respondents were queried about the frequency, attendance of MDTB members and available resources, and finally, their views on MDTBs using a 5-point Likert scale.

\subsection{Statistical analysis}

Data analysis was performed using SPSS v.19 (IBM, Armonk NY). Descriptive data was expressed as mean \pm standard deviation (SD) unless otherwise stated. One-way ANOVA was used for analysis of normallydistributed variables. Kruskal-Wallis test was used for non-normally distributed data. Categorical data was analyzed using Chi-square or Fisher's exact test. A value of $\mathrm{P}<0.05$ was considered statistically significant. Likert scale scores were summarized as ordinal approximations of a continuous measure $e^{26,27}$.

\section{Results}

Nine of 11 Southeast Asian countries were categorised as LMICs, representing 662,332,000 of 668,620,000 (99.1\%) of the total estimated population of Southeast Asia; of which 167,429,000 (25.0\%) were aged 14 years and under (Table 1$)^{28}$.

\subsection{Profile of PSTUs in Southeast Asia}

We identified 46 PSTUs across 9 Southeast Asian LMICs. Availability of MDTBs and specialty expertise could be established in 37 (80.4\%) PSTUs, and are summarized in Figure 1A. Among them 24 (52.2\%) PSTUs in 6 countries declared that they had regular MDTBs; PSTUs from Cambodia, Laos and Timor-Leste either did not have MDTBs or could not be contacted.

\subsection{Availability of subspecialty expertise in PSTUs}


Pediatric-trained oncologists, surgeons, radiologists, pathologists, radiation oncologists, nuclear medicine physicians and nurses were available in $94.6 \%, 91.9 \%, 54.1 \%, 40.5 \%, 29.7 \%, 13.5 \%$ and $81.1 \%$ of PSTUs, respectively (Fig 1B and Supplemental Table S1). Availability of pediatric-trained surgeons, radiologists and pathologists was significantly associated with existence of MDTBs $(p=0.037,0.005,0.022$, respectively) (Table 2).

\subsection{Profile of respondents}

Among the pairs of pediatric oncologists and pediatric surgeons contacted at each of the 24 PSTUs with MDTBs, 43 (89.6\%) individuals responded to the survey (21 pediatric oncologists and 22 pediatric surgeons). All respondents were pediatric trained. The oncology-dedicated workload was reported to be more than $50 \%$ in $90.5 \%$ of the oncologists versus only $22.7 \%$ among surgeons. Most respondents had more than 10 years' practice experience (61.9\% oncologists, $77.3 \%$ surgeons) (Supplemental Figure S1 and Supplemental Table S2).

\subsection{Profile of MDTBs among PSTUs in Southeast Asia}

Among the 24 PSTUs with MDTBs, oncologists, surgeons and radiologists were the most consistent attendees (Fig 2A). PSTUs most commonly conducted MDTBs once in a month (11 PSTUs (45.8\%)) (Fig 2A). The resources most commonly unavailable were facilities to view pathology slides before meeting and project them during the meeting (Fig 2B). Notably, 16 (37.2\%) respondents reported that there was either no allocated time limit for the meeting or were unsure if this was defined for their MDTB; 13 (30.2\%) reported that there was either no designated MDTB coordinator or were unsure (Fig 2B).

\subsection{Views on MDTB-related issues}

Likert-scale responses to 28 of $30(93.3 \%)$ questions did not differ between oncologist and surgeon respondents $(p>0.05)$. Significantly different responses were noted to 2 questions on patients that should be discussed at MDTBs ("All new pediatric cancer patients should be discussed in detail", and "Patient preferences and social circumstances should always be commented on") ( $p=0.015$ and 0.009 , respectively). Details of responses are summarized in Fig 3 and Supplemental Table S3.

\section{Discussion}

Southeast Asia is home to approximately 168 million children aged below 14 years, constituting one-fourth of its total population. At an estimated incidence of 92 cases per million, the region sees an estimated 16000 new cases of childhood cancer per annum - a disproportionate $9.6 \%$ of the global pediatric cancer burden ${ }^{29}$. Childhood cancer care and control programs in the region are still lacking, however substantial progress has been made in recent years, particularly via development of cooperative group structures ${ }^{29}$. We found that most countries in Southeast Asia have developed childhood cancer referral centers (Fig 1A), the majority of which are staffed by at least a dedicated pediatric-trained oncologist (Fig 1B). This is a tangible result of directed efforts in the field of pediatric oncology in Southeast Asia involving bodies and initiatives such as SIOP, St Jude Global, WHO Global Initiative for Childhood Cancer, VIVA Foundation for Children with Cancer, Southeast Asia Pediatric Hematology Oncology (SEAPHO), and Asian Children's Care League ${ }^{29}$. Such initiatives have included establishment of national pediatric cancer programs, education and training of the pediatric cancer healthcare workforce, and development of adapted-therapy treatment protocols. This demonstrates the impact of international partnerships in advocating for increased attention toward childhood cancer care as a global health priority ${ }^{30}$.

Pediatric solid tumor care is typically centered in referral centers and depends on the level of individual subspecialty capabilities and their coordination within multidisciplinary teams ${ }^{17}$. Presenting symptoms of pediatric solid tumors are more easily recognized than leukemias and brain tumors ${ }^{31-33}$, however their diversity of histological types and anatomical locations pose additional challenges to their successful management. Pediatric oncologists, the usual leaders of multidisciplinary solid tumor teams, need to collaborate with surgeons, radiologists, pathologists, radiation oncologists, nuclear medicine physicians and nurses. In 
this survey, we found only 4 of 46 PSTUs had pediatric-trained expertise in all 6 key subspecialities, with the rest supported mostly by general specialists. Despite this, 24 PSTUs could still organize regular MDTBs.

Pediatric surgeons were available in $91 \%$ of the PSTUs studied, the next most prevalent group of specialists after pediatric oncologists. Surgeons play an important role particularly in aspects of local control as well as venous access for chemotherapy. Interestingly, our survey found that the oncology-dedicated workload among pediatric surgeons was much lower than pediatric oncologists $(22.7 \%$ vs. $90.5 \%)$. Correspondingly, most were general pediatric surgeons without oncology-specific training. This reflects a very small numbers of centers in the region capable of providing level 3 surgical expertise with dedicated pediatric oncology surgeons ${ }^{17}$. Notably, expert groups have identified that pediatric surgery is a less recognized priority in global health, with less-established efforts to date that have focused on development of the speciality in LMICs ${ }^{34-36}$. Radiologists and pathologists play a significant role in diagnostic planning and recommendations. Only half of PSTUs were staffed with pediatric-trained radiologists and pathologists. The availability of these specialists in PSTUs was significantly associated with increased incidence of pediatric MDTBs. Correspondingly, the most acute gaps in multidisciplinary solid tumor care in this region were identified to be in the areas of radiographic and pathological diagnostic support (Fig 3D). The numbers of radiation oncologists and nuclear medicine physicians were even lower, particularly when considering pediatric-trained numbers. This highlights the manpower challenges faced by PSTUs, in addition to issues of availability of essential chemotherapy, surgery and basic diagnostic modalities.

Effective MDTBs require members' commitment to meet regularly as part of their recognized clinical duties, prepare and present required information and openly deliberate treatment recommendations in an evidence-based manner ${ }^{23}$. In limited resources settings especially in LMICs, organizing MDTBs can be an organizational burden and amounts to extra workload for the involved personnel ${ }^{23,24}$. Half of respondents reported that "lack of time" and "too much workload to attend the meeting regularly" were among the main barriers they faced personally. Notably, we observed that most MDTBs shared common views on ideal goals and factors for success, and that oncologist and surgeons' opinions did not differ significantly, particularly on workflow-related matters such as prioritization of cases for discussion and tangible benefits for PSTU teams. Most MDTBs had necessary infrastructure such as meeting venues and access to radiology images before and during the meeting. Interestingly, organizational challenges appeared to be a common problem. Despite most respondents ranking the need for clear guidelines and pre-meeting agendas highly, only about $70 \%$ of respondents reported having a designated coordinator and circulation of pre-meeting agendas and patient lists. This points to an underlying lack of support systems among pediatric cancer units in Southeast Asia - a gap which likely also accounts for the observed lack of registry data from centers in this region ${ }^{31,33,37}$.

Delivery of care for pediatric oncology patients is also impacted by social, economic and cultural factors. While the formation of an MDTB is a first essential step for PSTUs to ensure correct diagnoses and proper treatment recommendations, obstacles to childhood cancer care faced by LMICs extend beyond this. Globally, there are significant gaps in the distribution of financial resources for pediatric cancer care: expenditure in LMICs amount to only $6.2 \%$ of global spending, yet they care for a disproportionate two-thirds of childhood cancer cases worldwide ${ }^{29}$. While over the previous decade, Southeast Asian countries received significantly less funding from international grants (31), such support has increased in recent years (32). Yet, our data demonstrates how imbalanced resource distribution in Southeast Asia impacts childhood cancer care in highly populous and lower income countries. Southeast Asian LMICs with higher GNI per capita had more pediatric solid tumor MDTBs, particularly Malaysia and Thailand, with the 2 highest GNIs per capita (Fig 1). However MDTB frequency did not correspond with the size of national pediatric populations: Indonesia, Philippines and Myanmar have $70 \%$ of the children under 14 years in Southeast Asia but only $40 \%$ of the MDTBs. All 3 countries have a GNIs per capita below US $\$ 4000$. Additionally, the diversity of ethnicity, language, religion and culture in Southeast Asia add to the challenge of health equity. Also, availability of essential medicines, abandonment and local socio-cultural nuances such as use of traditional medicines are yet more challenges, all of which are under-studied in the Southeast Asian region ${ }^{38,39}$.

This study was limited by the scope of coverage of PSTUs in the Southeast Asian countries, with some being 
inadvertently missed, and some not responding to the survey. Nevertheless, the $80.4 \%$ of PSTUs profiled represent at least each of the main national referral centers in the region, most fulfilling criteria as Level 2 Pediatric Cancer Units (PCUs), according to the SIOP PODC framework ${ }^{17}$. It can be reasonably expected that centers not covered by this study would be PSTUs with Level 1 facilities especially from lower GNI countries such as Cambodia, Laos and Timor Leste, and more populous or geographically larger countries such as Indonesia, Philippines and Vietnam. This study may also over-represent the pediatric solid tumor capabilities of the region. Because of the heterogeneity of training models in various countries, no specific definitions were imposed to differentiate between "pediatric-trained" or "general" specialists, and this was left to individual respondents' interpretation. In cases of discrepant responses between oncologists and surgeons, the higher level of expertise was taken to represent the center, given the liberal definition applied. Even then, most PSTUs lacked pediatric-trained pathologists and radiologists, as well as radiation oncologists and nuclear medicine physicians. These numbers would be expected to be even lower in level 1 PCUs which were not covered in this study. Notably, other surgical subspecialties involved such as ophthalmologists and orthopedic surgeons were also not profiled in this survey.

From this study, we propose several recommendations to develop PSTUs and MDTBs to improve pediatric solid tumor care in Southeast Asia:

1. Development of multidisciplinary teams. LMIC PSTU teams may benefit from intentional exposure and modelling from established PTSUs. Adapted systematic recommendations could be proposed to guide team development and constitution, and MDTB execution, including best practices for premeeting preparation, documentation of proceedings and self-auditing ${ }^{40,41}$.

2. Optimization of local MDTB administration. PSTUs may benefit from improved organization of MDTB meetings. Increased involvement of non-clinical staff or nurses may help to overcome workload and time limitations faced by clinicians. Recognizing MDTBs as a professional activity with incentives for attendance such as CPD points may further increase participation.

3. Expansion and ongoing support for regional training resources. Pediatric oncology training programs and collaborations in Southeast Asia that have come about as a result of recent nongovernmental organizations' support should be continued and widened now to include and develop more specialties, especially pediatric surgery, radiology and pathology, with enhanced support from governmental bodies and international charities.

\section{Conclusions}

This cross-sectional survey highlighted the current availability of essential specialty expertise and MDTB structures in most PSTUs in Southeast Asian countries. Recent regional initiatives and collaborations have been a clear contributor to these developments. However, the lack of pediatric-trained subspecialists, particularly dedicated pediatric oncology surgeons, and pediatric-trained pathologists and radiologists, remains a gap in the workforce required for capable multidisciplinary care of solid tumors. An extended spectrum of training programs is needed to focus on these subspecialties as well.

\section{Funding and Conflicts of Interest}

This study was supported by the VIVA Foundation for Children with Cancer (A.H.P.L., M.Y.O., VIVA-KKH Paediatric Brain and Solid Tumour Programme). Authors declare no other conflicts of interest.

\section{Acknowledgements}

The authors thank all survey participants from Phnom Penh and Siem Reap, Cambodia; Bandung, Jakarta, Medan, Manado, Surabaya, and Yogjakarta, Indonesia; Ipoh, Kota Kinabalu, Kuala Lumpur, Kuching, and Penang, Malaysia; Mandalay, Yangon, and Yankin, Myanmar; Batangas, Davao City and Manila, Philippines; Bangkok, Chiang Mai, and Songkla, Thailand; and Hanoi, Ho Chi Minh City, and Hue, Vietnam.

References

1. Tan AM, Ha C, Li CF, et al. Viva-Asia Blood and Marrow Transplantation Groups - A Survey of 
Consortium Activity over a 12-year Period (2000 to 2011). Ann Acad Med Singapore.2016;45(3):106-109.

2. Ali K, Sutaryo S, Purwanto I, et al. Yogyakarta Pediatric Cancer Registry: an international collaborative project of University Gadjah Mada, University of Saskatchewan, and the Saskatchewan Cancer Agency.Asian Pac J Cancer Prev. 2010;11(1):131-136.

3. Park KD, Hong CR, Choi JY, et al. Foundation of pediatric cancer treatment in Lao People's Democratic Republic at the Lao-Korea National Children's Hospital. Pediatr Hematol Oncol. 2018;35(4):268-275.

4. Halbert J, Khaing AA. Overview of pediatric oncology and hematology in Myanmar. South Asian $J$ Cancer. 2014;3(1):78-82.

5. Bidwell SS, Peterson CC, Demanelis K, et al. Childhood cancer incidence and survival in Thailand: A comprehensive population-based registry analysis, 1990-2011. Pediatr Blood Cancer.2019;66(1):e27428.

6. Ward ZJ, Yeh JM, Bhakta N, Frazier AL, Atun R. Estimating the total incidence of global childhood cancer: a simulation-based analysis.Lancet Oncol. 2019;20(4):483-493.

7. GLOBOCAN 2012: Estimated Cancer Incidence, Mortality and Prevalence Worldwide in 2012. http://globocan.iarc.fr/Pages/age-specific_table_sel.aspx.

8. International Incidence of Childhood Cancer, Volume III (electronic version). International Agency for Research on Cancer; 2017. http://iicc.iarc.fr/results/. Accessed 14 Mar 2020.

9. Rajagopal R, Abd-Ghafar S, Ganesan D, et al. Challenges of Treating Childhood Medulloblastoma in a Country With Limited Resources: 20 Years of Experience at a Single Tertiary Center in Malaysia. J Glob Oncol. 2017;3(2):143-156.

10. Sangkhathat S, Chotsampancharaen T, Kayasut K, et al. Outcomes of pediatric nephroblastoma in southern Thailand. Asian Pac J Cancer Prev. 2008;9(4):643-647.

11. Fabian ID, Abdallah E, Abdullahi SU, et al. Global Retinoblastoma Presentation and Analysis by National Income Level. JAMA Oncol.2020.

12. Adham M, Stoker SD, Wildeman MA, et al. Current status of cancer care for young patients with nasopharyngeal carcinoma in Jakarta, Indonesia. PLoS One. 2014;9(7):e102353.

13. Wongmas P, Jetsrisuparb A, Komvilaisak P, et al. Incidences, Trends and Long Term Outcomes of Retinoblastoma in Three Cancer Registries, Thailand. Asian Pac J Cancer Prev. 2015;16(16):6899-6902.

14. Seksarn P, Wiangnon S, Veerakul G, Chotsampancharoen T, Kanjanapongkul S, Chainansamit SO. Outcome of Childhood Acute Lymphoblastic Leukemia Treated Using the Thai National Protocols.Asian Pac J Cancer Prev. 2015;16(11):4609-4614.

15. Wongmeerit P, Suwanrungruang K, Jetsrisuparb A, Komvilaisak P, Wiangnon S. Trends in Survival of Childhood Cancers in a University Hospital, Northeast Thailand, 19932012. Asian Pac J Cancer Prev.2016;17(7):3515-3519.

16. Tah PC, Nik Shanita S, Poh BK. Nutritional status among pediatric cancer patients: a comparison between hematological malignancies and solid tumors. J Spec Pediatr Nurs. 2012;17(4):301-311.

17. Howard SC, Davidson A, Luna-Fineman S, et al. A framework to develop adapted treatment regimens to manage pediatric cancer in low- and middle-income countries: The Pediatric Oncology in Developing Countries (PODC) Committee of the International Pediatric Oncology Society (SIOP).Pediatr Blood Cancer. 2017;64 Suppl 5.

18. Madani A, Zafad S, Harif M, et al. Treatment of Wilms tumor according to SIOP 9 protocol in Casablanca, Morocco. Pediatr Blood Cancer. 2006;46(4):472-475. 
19. Pedrosa F, Bonilla M, Liu A, et al. Effect of malnutrition at the time of diagnosis on the survival of children treated for cancer in El Salvador and Northern Brazil. J Pediatr Hematol Oncol.2000;22(6):502-505.

20. Kreyer J, Ranft A, Timmermann B, et al. Impact of the Interdisciplinary Tumor Board of the Cooperative Ewing Sarcoma Study Group on local therapy and overall survival of Ewing sarcoma patients after induction therapy. Pediatr Blood Cancer.2018;65(12):e27384.

21. George PE, Fahdil G, Luutu I, et al. Analysis of management decisions and outcomes of a weekly multidisciplinary pediatric tumor board meeting in Uganda. Future Sci OA. 2019;5(9):FSO417.

22. Thenappan A, Halaweish I, Mody RJ, et al. Review at a multidisciplinary tumor board impacts critical management decisions of pediatric patients with cancer. Pediatr Blood Cancer.2017;64(2):254-258.

23. Rosell L, Alexandersson N, Hagberg O, Nilbert M. Benefits, barriers and opinions on multidisciplinary team meetings: a survey in Swedish cancer care. BMC Health Serv Res. 2018;18(1):249.

24. Shulman T, Bain CA, Raikundalia GK, Sharma RK. Obstacles to Sustaining Cancer Care Multidisciplinary Team Meetings: An Australian Survey. 2013.

25. Taylor CT, Ramirez AR. Multidisciplinary team members' views about MDT working: Results from a survey commissioned by the National Cancer Action Team. In. London SE1 7EH: Cancer Research UK Promoting Early Presentation Group; 2009.

26. Norman G. Likert scales, levels of measurement and the "laws" of statistics. Adv Health Sci Educ Theory Pract. 2010;15(5):625-632.

27. Sullivan GM, Artino AR. Analyzing and interpreting data from likert-type scales. J Grad Med Educ. 2013;5(4):541-542.

28. Nations U. World Population Prospects 2019: Data Booket. ST/ESA/SER.A/424. In: Department of Economic and Social Affairs, Population Division: https://population.un.org/wpp/Publications/Files/WPP2019_DataBooklet.pdf.

29. Rodriguez-Galindo C, Friedrich P, Alcasabas P, et al. Toward the Cure of All Children With Cancer Through Collaborative Efforts: Pediatric Oncology As a Global Challenge. J Clin Oncol.2015;33(27):30653073.

30. Rodriguez-Galindo C, Friedrich P, Morrissey L, Frazier L. Global challenges in pediatric oncology. Curr Opin Pediatr.2013;25(1):3-15.

31. Howard SC, Metzger ML, Wilimas JA, et al. Childhood cancer epidemiology in low-income countries. Cancer.2008;112(3):461-472.

32. Parkin DM, Stiller CA, Draper GJ, Bieber CA. The international incidence of childhood cancer. Int J Cancer. 1988;42(4):511-520.

33. G upta S, Howard SC, Hunger SP, et al. Treating Childhood Cancer in Low- and Middle-Income Countries. In: Gelband H JP, Sankaranarayanan R, et al., ed. Cancer: Disease Control Priorities, Third Edition.Vol 3. Washington (DC): The International Bank for Reconstruction and Development / The World Bank; 2015.

34. Bickler SW, Rode H. Surgical services for children in developing countries. Bull World Health Organ. 2002;80(10):829-835.

35. Surgery GIfCs. Global Initiative for Children's Surgery: A Model of Global Collaboration to Advance the Surgical Care of Children. World J Surg. 2019;43(6):1416-1425.

36. Goodman LF, St-Louis E, Yousef Y, et al. The Global Initiative for Children's Surgery: Optimal Resources for Improving Care. Eur J Pediatr Surg. 2018;28(1):51-59. 
37. Moore MA, Shin HR, Curado MP, Sobue T. Establishment of an Asian Cancer Registry Network problems and perspectives. Asian Pac J Cancer Prev. 2008;9(4):815-832.

38. Hamidah A, Rustam ZA, Tamil AM, Zarina LA, Zulkifli ZS, Jamal R. Prevalence and parental perceptions of complementary and alternative medicine use by children with cancer in a multi-ethnic Southeast Asian population. Pediatr Blood Cancer. 2009;52(1):70-74.

39. Mostert S, Gunawan S, van Dongen JA, et al. Health-care providers' perspectives on childhood cancer treatment in Manado, Indonesia.Psychooncology. 2013;22(11):2522-2528.

40. Standards for Pediatric Cancer Centers. Pediatrics.2014;134(2):410-414.

41. Borras JM, Albreht T, Audisio R, et al. Policy statement on multidisciplinary cancer care. Eur J Cancer. 2014;50(3):475-480.

Figure legends

FIGURE 1 Distribution of the multidisciplinary workforce caring for pediatric solid tumors in Southeast Asia. (A) Geographical locations of PCUs in Southeast Asia with indication of level of expertise available for 7 key roles (oncologists, surgeons, radiologists, pathologists, radiation oncologists, nuclear medicine physicians and nurses) in each center, against the percentage of centers per country with MDTBs. (B) Total number of personnel and proportion of corresponding levels of expertise for 7 key roles, for 9 Southeast Asian countries, ranked by size of national population aged 19 and under (United Nations, Department of Economic and Social Affairs, Population Division (2019). World Population Prospects 2019.)

FIGURE 2 Profile of pediatric MDTBs in Southeast Asia. (A) Reported average attendance of 7 key roles (oncologists, surgeons, radiologists, pathologists, radiation oncologists, nuclear medicine physicians and nurses) at MDTBs, and reported frequency of MDTB meetings in 24 centers in 6 Southeast Asian countries. (B) Availability of MDTB resources as reported by oncologists and surgeons.

FIGURE 3 Views on pediatric MDTBs. Views of oncologists and surgeons from 6 Southeast Asian countries towards (A) factors that make for effective MDTBs, (B) benefits experienced by MDTBs, (C) patients being discussed at MDTBs, and (D) barriers faced by MDTBs in their centers.

Supplemental FIGURE S1 Profile of respondents (21 pediatric oncologists and 22 pediatric surgeons) and their level of training, proportion of workload dedicated to oncology patients and years in practice.

\section{Hosted file}

Table 1.docx available at https://authorea.com/users/320021/articles/449651-profile-ofpediatric-solid-tumor-care-and-multidisciplinary-tumor-boards-in-southeast-asia

\section{Hosted file}

Table 2.docx available at https://authorea.com/users/320021/articles/449651-profile-ofpediatric-solid-tumor-care-and-multidisciplinary-tumor-boards-in-southeast-asia 


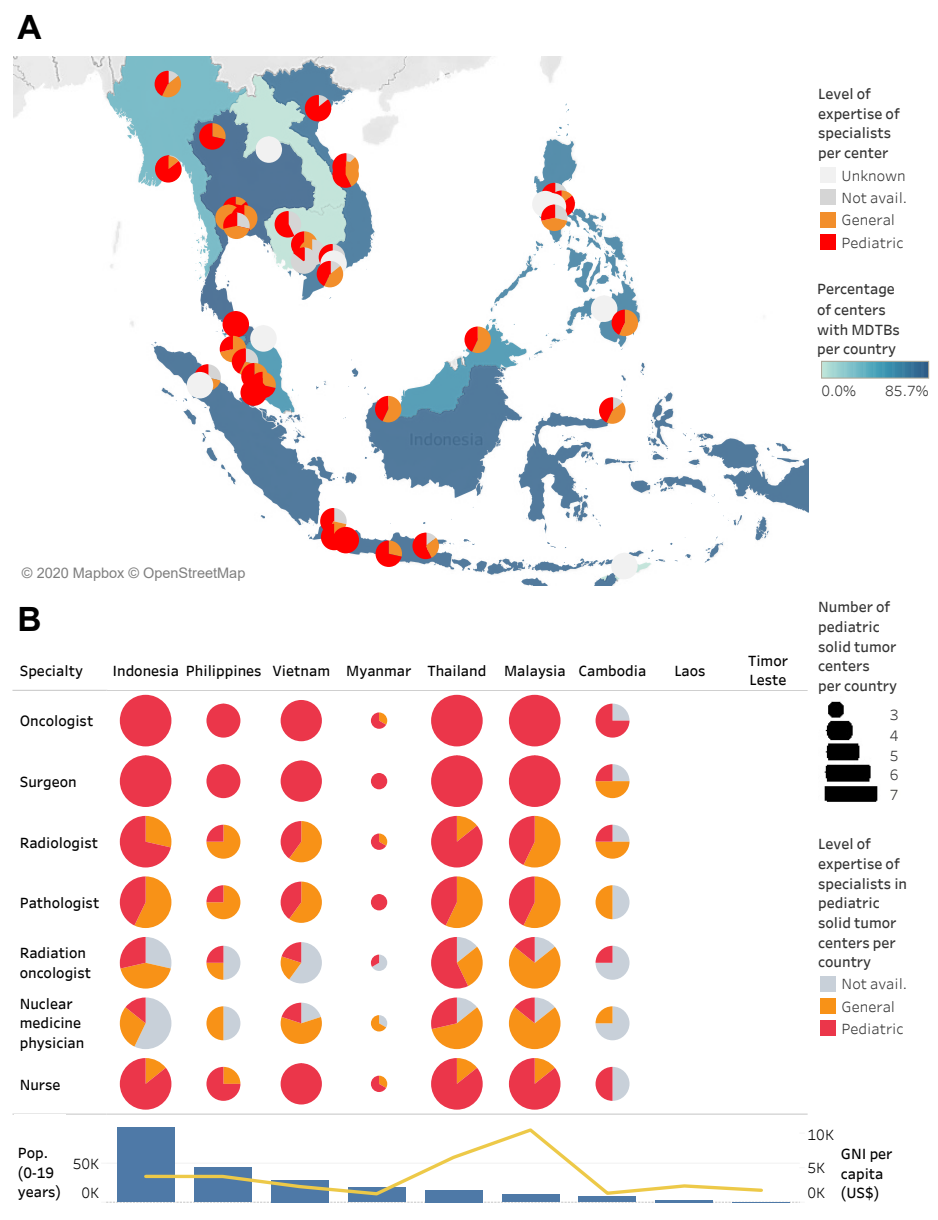


A

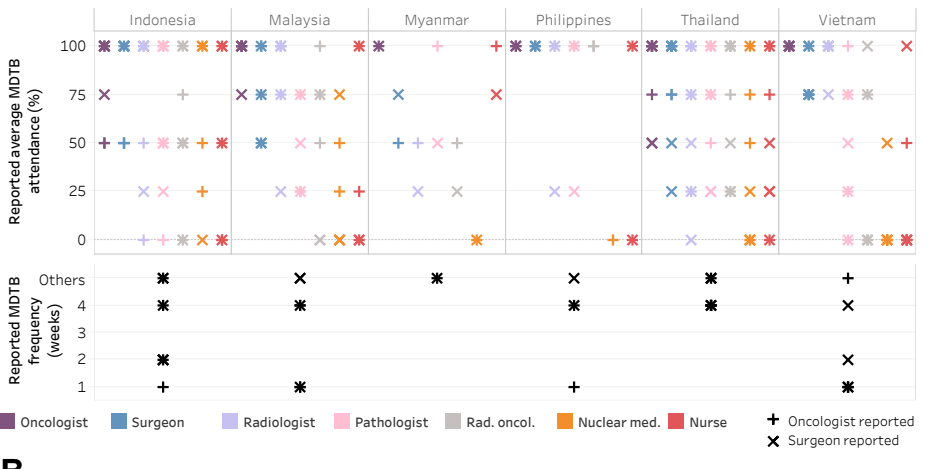

B

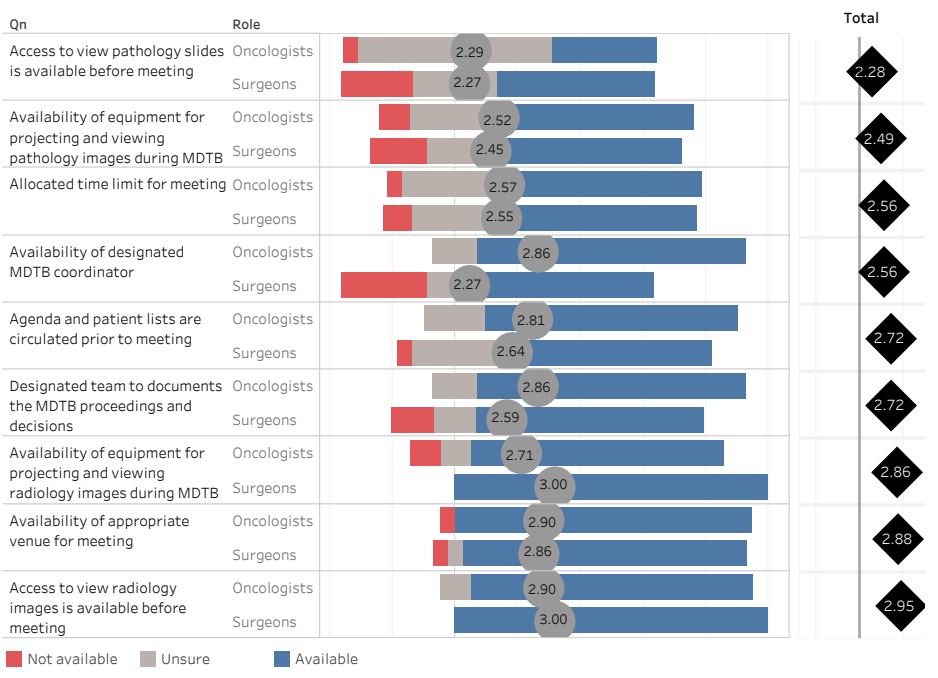


A Views on factors that make for an effective MDTB
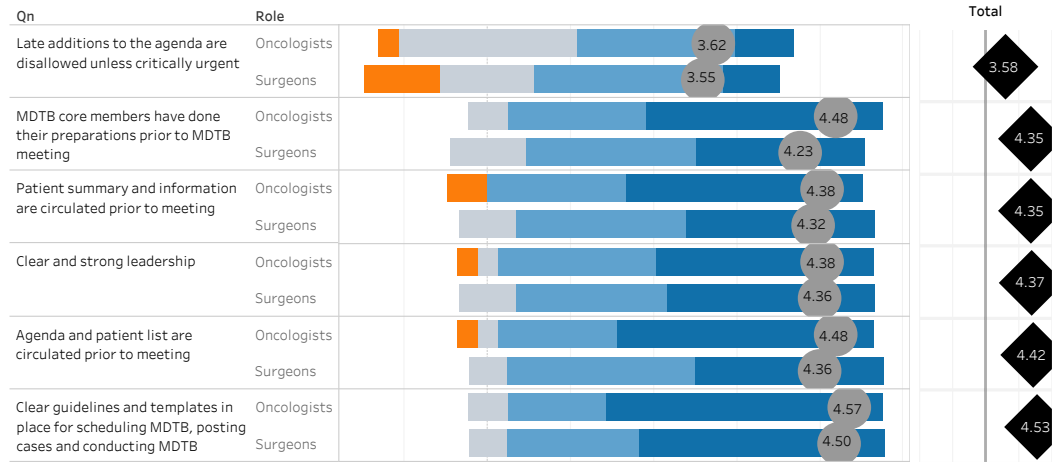

B Views on benefits experienced by MDTBs
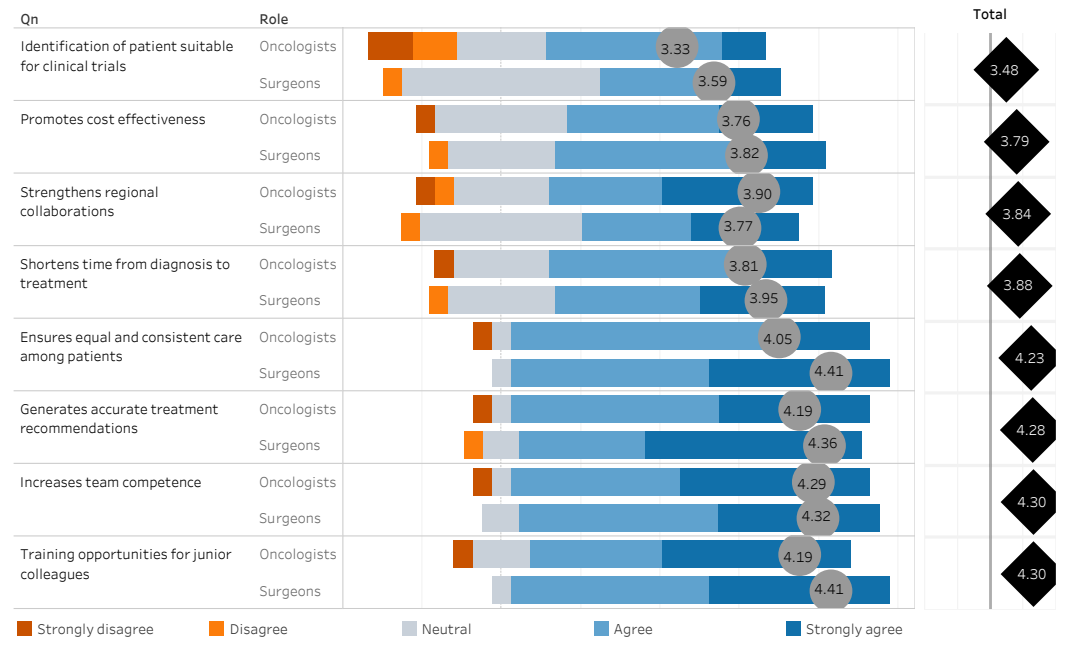